\title{
An Evolutionary Model for Collapsing Molecular Clouds and Their Star Formation Activity. II. Mass Dependence of the Star Formation Rate
}

\author{
Manuel Zamora-Avilés ${ }^{1} \&$ Enrique Vázquez-Semadeni ${ }^{1}$
}

Received —

Submitted to ApJ

${ }^{1}$ Centro de Radioastronomía y Astrofísica, Universidad Nacional Autónoma de México, Apdo. Postal 3-72, Morelia, Michoacán, 58089, México 


\begin{abstract}
We discuss the evolution, and dependence on cloud mass, of the star formation rate (SFR) and efficiency (SFE) of star-forming molecular clouds (MCs), within the scenario that clouds are undergoing global collapse, and that the SFR is controlled by ionization feedback. We find that low-mass clouds $\left(M_{\max } \lesssim 10^{4} \mathrm{M}_{\odot}\right)$ spend most of their evolution at low SFRs, but end their lives with a mini-burst, reaching a peak $\mathrm{SFR} \sim 10^{4} \mathrm{M}_{\odot} \mathrm{Myr}^{-1}$, although their time-averaged SFR is only $\langle\mathrm{SFR}\rangle \sim 10^{2} \mathrm{M}_{\odot} \mathrm{Myr}^{-1}$. The corresponding efficiencies are $\mathrm{SFE}_{\text {final }} \lesssim 60 \%$ and $\langle\mathrm{SFE}\rangle \lesssim 1 \%$. For more massive clouds $\left(M_{\max } \gtrsim 10^{5} \mathrm{M}_{\odot}\right)$, the SFR first increases and then reaches a plateau, because the clouds are influenced by the stellar feedback since earlier in their evolution. As a function of cloud mass, $\langle\mathrm{SFR}\rangle$ and $\langle\mathrm{SFE}\rangle$ are well represented by the fits $\langle\mathrm{SFR}\rangle \approx 100\left(1+M_{\max } / 1.4 \times\right.$ $\left.10^{5} \mathrm{M}_{\odot}\right)^{1.68} \mathrm{M}_{\odot} \mathrm{Myr}^{-1}$ and $\langle\mathrm{SFE}\rangle \approx 0.03\left(M_{\max } / 2.5 \times 10^{5} \mathrm{M}_{\odot}\right)^{0.33}$, respectively. Moreover, the SFR of our model clouds follows closely the SFR-dense gas mass relation recently found by Lada et al., during the epoch when their instantaneous SFEs are comparable to those of the clouds considered by those authors. Collectively, a Monte Carlo integration of the model-predicted $\operatorname{SFR}(M)$ over a Galactic GMC mass spectrum yields values for the total Galactic SFR that are within half an order of magnitude from the relation obtained by Gao \& Solomon. Our results support the scenario that star-forming MCs may be in global gravitational collapse, and that the low observed values of the SFR and SFE are a result of the interruption of each SF episode, caused primarily by the ionizing feedback from massive stars.
\end{abstract}

Subject headings: ISM: clouds — ISM: evolution — Stars: formation 


\section{Introduction}

The regulation of the star formation rate (SFR) in molecular clouds (MCs) has been a key problem in astrophysics for over half a century, ever since Schmidt (1959) noticed that the SFR in clouds exhibited a power-law dependence on the gas number density $n$. A crucial aspect of the SFR was noticed by Zuckerman \& Palmer (1974), who pointed out that the observed Galactic SFR is at least one order of magnitude lower than that expected if the clouds were forming stars at the "free-fall rate", given by the ratio of the total molecular gas mass in the Galaxy to the typical free-fall time of this gas. Indeed, current estimates of the total molecular gas mass and density $\left(M_{\text {mol }} \sim 10^{9} \mathrm{M}_{\odot}, n \sim 100 \mathrm{~cm}^{-3}\right.$; e.g., Ferrière 2001) imply a free-fall SFR $\sim 200 \mathrm{M}_{\odot} \mathrm{yr}^{-1}$, while the observed SFR is roughly 100 times smaller (e.g., Chomiuk \& Povich 2011). Thus, it was concluded that MCs could not be in free-fall, contrary to the then recent suggestion of Goldreich \& Kwan (1974), and that the nonthermal linewidths observed in the clouds were produced by small-scale turbulence instead (Zuckerman \& Evans 1974).

Since then, MCs have been assumed to be supported by a number of physical agents, such as magnetic fields (e.g., Shu et al. 1987; Mouschovias 1991) or turbulence (e.g., Vázquez-Semadeni et al. 2000; Vázquez-Semadeni et al. 2003; Mac Low \& Klessen 2004; Elmegreen \& Scalo 2004; Ballesteros-Paredes et al. 2007; McKee \& Ostriker 2007). In both scenarios, the necessary low SFR was attained because a small fraction of the mass managed to escape the support. This fraction was mediated by ambipolar diffusion in the first case, and by local turbulent compressions which induced small-scale, low-mass collapses in the second. In the last decade, a number of models for the turbulent regulation of star formation (SF) have been constructed within the scenario of clouds in which both the global support and the local collapses are induced by turbulence (Krumholz \& McKee 2005; Padoan \& Nordlund 2011; Hennebelle \& Chabrier 2011). These models are based on 
the premise that the high-density tail of the density probability density function (PDF), which takes a lognormal form for supersonic isothermal turbulence (Vázquez-Semadeni 1994; Padoan et al. 1997; Passot \& Vázquez-Semadeni 1998), is responsible for the instantaneous SFR, which is given by this mass, divided by a characteristic timescale. The models differ in how the threshold density for defining the "high-density" gas and the characteristic timescale. A thorough discussion of these models has been recently provided by Federrath \& Klessen (2012).

However, recent evidence from both observations and numerical simulations has suggested that star-forming MCs may be in gravitational collapse after all. Comparing numerical simulations of a variety of turbulent and free-falling regimes to the observed kinematics of the clump NGC 2264-C, Peretto, Hennebelle \& André (2007) showed that the best fit was provided by simulations in which infall dominates over turbulence by a large margin (95\% of the kinetic energy). Comparing the morphology of the Orion A cloud to that of simulations of gravitational collapse of a nearly elliptical sheet of gas, Hartmann \& Burkert (2007) suggested that the entire Orion A cloud may be in gravitational collapse. Also, infall has been observed at multiple scales in the high-mass star forming region G20.08-0.14 (Galván-Madrid et al. 2009) and from filamentary regions onto clumps, as well as onto the filaments (Schneider et al. 2010; Kirk et al. 2013). On the numerical side, simulations of cold, dense cloud formation including self-gravity (Vázquez-Semadeni et al. 2007, 2009, 2010, 2011; Heitsch \& Hartmann 2008) have shown that the clouds engage in gravitational collapse shortly after they collect enough mass to be Jeans-unstable, and long before any star formation begins to occur within them. Moreover, the nonlinear density fluctuations produced by the turbulence in the cloud have shorter free-fall timescales than the cloud at large, and therefore complete their collapses before the cloud does. Thus, Vázquez-Semadeni et al. (2009) suggested that MCs are in a state of "hierarchical gravitational collapse", where the local, small-scale collapses of dense cores are occurring 
within the global, large-scale collapse of the cloud.

In a previous paper (Zamora-Avilés, Vázquez-Semadeni \& Colín 2012, hereafter Paper I), we presented an analytical model for the evolution of the SFR in the context of gravitationally collapsing clouds. This model was based on the same prescription for computing the SFR as that used in the models mentioned above; i.e., an integration of the density PDF above a certain threshold density to obtain the mass responsible for the "instantaneous" SFR. The threshold density was obtained by calibrating the evolution of the SFR with the numerical simulations of Vázquez-Semadeni et al. (2010), and the timescale was chosen as the free-fall time at the threshold density. However, the distinctive feature of the model was that the cloud, assumed to have a sheet-like geometry, was considered to be undergoing free-fall gravitational collapse, causing its mean density to increase. Therefore, the density PDF was considered to continuously shift to higher densities, and thus the star-forming mass continuously increased in time, implying a systematic increase of the SFR. The controlling parameter of the model was found to be the total system mass, and the model successfully described the evolutionary sequence for $\sim 10^{5} \mathrm{M}_{\odot}$ GMCs reported by Kawamura et al. (2009), the stellar age histograms for clouds of mass $\sim 2000 \mathrm{M}_{\odot}$, as reported by Palla \& Stahler (2000, 2002), and the locus of clouds of this same mass in the Kennicutt-Schmidt diagram, as reported by Evans et al. (2009).

Since the main controlling parameter of the model from Paper I was the cloud's mass, in this paper we now examine the predictions of the model for the dependence of the SFR and the star formation efficiency (SFE) with the mass of the cloud, and from there examine the prediction of the model for the SFR-mass relation first proposed by Gao \& Solomon (2004, hereafter, the GS relation). The plan of the paper is as follows: in Sec. 2, we present a brief summary of the model, as well as its application to the present study. In Sec. 3 we present the results for the dependence of the SFR and the SFE with cloud mass, and 
compare the model with the observational GS relation. Then, in Sec. 4 we discuss some implications and limitations of our results. Finally, in Sec. 5 we present a summary.

\section{The Model}

Our model, first presented in Paper I, follows the evolution of the gas mass that initially constitutes a cold atomic cloud, formed by the collision of two streams in the warm neutral medium (WNM). The model is intermediate between a Lagrangian and an Eulerian description, as it follows the collapse of the cold cloud material as soon as it exceeds its Jeans mass, but at the same time allows for the addition of fresh material, coming from the continuing WNM streams ("the inflows"), through the boundaries of the collapsing region. As the cold gas collapses and reduces its size, we only add to it the material entering through its instantaneous, reduced boundaries, while the rest of the inflow material is assumed to be deposited in an envelope, whose evolution we ignore. Thus, the model accounts for the fact that a "cloud" is not made of the same material throughout its evolution, but rather is constantly accreting fresh material from its environment, as has been proposed by several studies (e.g., Vázquez-Semadeni et al. 2009, 2010; Smith et al. 2009; Goldbaum et al. 2011). Within this scenario, we follow the evolution of the material that initially begins to collapse, to which we will, for convenience, refer to as "the cloud", although it must be borne in mind that the entire system consists of this collapsing region plus the material added to the envelope during the evolution. Thus, the entire system does not contract because of the material continuously added to it.

We assume that the clouds are born with a density of $n=100 \mathrm{~cm}^{-3}$ and a 
temperature of $\sim 40 \mathrm{~K}, 1$ representative of the cold atomic medium (CNM). In Paper I, the flows were assumed to continue indefinitely, as is done in many numerical simulations (e.g., Audit \& Hennebelle 2005; Hennebelle et al. 2008). Instead, here we assume that the flows subside after $25 \mathrm{Myr}$, somehow mimicking the duration of the accretion flow that a parcel in the ISM may be subject to when traversing a 1-kpc spiral arm at a speed of $\sim 20 \mathrm{~km} \mathrm{~s}^{-1}$ (the spiral pattern speed with respect to the gas at the Solar circle). The mass accretion rate onto the cloud is given by $\dot{M}_{\text {inf }}=2 \rho_{\mathrm{WNM}} v_{\text {inf }}\left(\pi R_{\text {inf }}^{2}\right)$, where $\rho_{\mathrm{WNM}}$ is the WNM density $\left(=2.1 \times 10^{-24} \mathrm{~g} \mathrm{~cm}^{-3}\right.$, which corresponds to $n=1 \mathrm{~cm}^{-3}$ assuming a mean particle mass of 1.27$), v_{\text {inf }}$ is the inflow velocity $\left(=4.5 \mathrm{~km} \mathrm{~s}^{-1}\right.$, obtained from the calibration; see below), and $R_{\text {inf }}$ is the radius of the inflow, assumed to have a cylindrical shape. This inflow continues to feed the cloud for $25 \mathrm{Myr}$, increasing its mass. We assume that the cloud begins to undergo global gravitational collapse as soon as it reaches its Jeans mass which, for a planar cloud, is given by (Larson 1985)

$$
M_{\mathrm{J}}=4.67 \frac{c_{\mathrm{s}}^{4}}{G^{2 \Sigma}}
$$

where $c_{\mathrm{s}}$ is the sound speed in the cloud, assumed constant and uniform $\left(c_{\mathrm{s}}(T=40 \mathrm{~K})=\right.$ $0.38 \mathrm{~km} \mathrm{~s}^{-1}$ ), and $\Sigma=M_{\mathrm{C}}(t) / \pi R_{\mathrm{C}}^{2}(t)$ is the surface density, with $M_{\mathrm{C}}(t)$ and $R_{\mathrm{C}}(t)$ being the instantaneous mass and radius of the cloud, respectively. Note that we deliberately do not consider turbulent support, as one essential feature of the model is that the large supersonic velocities that develop in molecular clouds are the result of the collapse, and thus do not provide support. We also assume that, because the initial turbulence is transonic (see below), it does not provide a significant source of additional support. Finally, note also that, once the cloud has started to collapse, its radius shrinks, and so we only consider the mass inflow across its instantaneous cross section, assuming that the rest of the material

\footnotetext{
${ }^{1}$ This temperature is obtained considering the heating and cooling processes by Koyama \& Inutsuka (2000).
} 
goes into a medium-density $\left(\sim 100 \mathrm{~cm}^{-3}\right)$ cloud envelope, which is not included in the collapse calculation.

We furthermore assume that the cold dense gas is turbulent, due to the combined action of various instabilities (Vishniad 1994; Walder \& Folini 2000; Heitsch et al. 2006; Vázquez-Semadeni et al. 2006), with a moderate Mach number $\mathcal{M}_{\text {rms }}=3$. This is consistent with observations of the velocity dispersion in the cold neutral medium (Heiles \& Troland 2003). Note that this Mach number is significantly lower than the typical Mach numbers, $\mathcal{M}_{\text {rms }} \sim 10-20$, usually associated with molecular clouds, which in our model correspond to infall velocities rather than to random turbulence. We stress that numerical simulations in general (Kovama \& Inutsuka 2002; Audit \& Hennebelle 2005; Vázquez-Semadeni et al. 2006, 2007; Banerjee et al. 2009) show that the turbulent Mach numbers produced in the atomic precursor of a MC by the flow collision are substantially smaller than those observed in MCs. As discussed in Vázquez-Semadeni et al. (2007), such high Mach numbers are only observed in cloud evolution simulations as a consequence of the gravitational contraction. As a consequence of the turbulence in the dense gas, we assume that the cloud develops a lognormal density PDF of the form

$$
P(s)=\frac{1}{\sqrt{2 \pi \sigma_{s}^{2}}} \exp \left[-\frac{\left(s-s_{\mathrm{p}}\right)^{2}}{2 \sigma_{s}^{2}}\right],
$$

where $s \equiv \ln (\rho /\langle\rho\rangle), s_{\mathrm{p}}=\ln \left(\rho_{\mathrm{p}} /\langle\rho\rangle\right)=-\sigma_{s}^{2} / 2$, and $\sigma_{s}^{2}=\ln \left(1+b^{2} \mathcal{M}_{\mathrm{rms}}^{2}\right)$, with $\rho_{\mathrm{p}}$ being the peak density, $\langle\rho\rangle$ the mean density, and $b$ a proportionality constant related to the compressibility induced by the turbulent forcing (Federrath et al. 2008, 2010). For simplicity, we consider only compressible modes, i.e. $b=1$.

As in other recent SFR models (Krumholz \& McKee 2005; Padoan \& Nordlund 2011; Hennebelle \& Chabrier 2011; Federrath \& Klessen 2012), we assume that the high-density tail of the PDF is responsible for the instantaneous SFR in the cloud, which is calculated as

$$
\operatorname{SFR}(t)=\frac{M\left(n>n_{\mathrm{SF}}, t\right)}{t_{\mathrm{ff}}\left(n_{\mathrm{SF}}\right)},
$$


where $n_{\mathrm{SF}}$ is a threshold number density for defining the mass involved in the instantaneous $\mathrm{SFR}$, and $t_{\mathrm{ff}}\left(n_{\mathrm{SF}}\right)$ is the free-fall time at number density $n_{\mathrm{SF}}$. Note that $n_{\mathrm{SF}}$ represents neither the mean density of the cloud nor the typical density of the clumps that form stars. Instead, it is a free parameter of the model indicating the density above which the collapse time can be considered to be negligibly small compared to the evolutionary timescale of the system. That is, since the cloud contains a distribution of density fluctuations caused by the turbulence, the densest among these have the shortest collapse timescales. The parameter $n_{\mathrm{SF}}$ represents the density fluctuation level whose collapse time can be considered as "instantaneous" in the model. On the other hand, the typical density of a star-forming cloud or clump is represented by the peak of the density PDF, and is generally smaller than $n_{\mathrm{SF}}$, except at the very last stages of the collapse of a model cloud, when its mean density reaches very high values (see below).

The threshold number density $n_{\mathrm{SF}}$ was calibrated in Paper I by matching the evolution of the model to the results of the numerical simulations -specifically, the evolution of the SFR, and the gaseous and stellar masses. The best match was found to occur for $n_{\mathrm{SF}}=10^{6} \mathrm{~cm}^{-3}$ (for which the free-fall time is $t_{\mathrm{ff}} \approx 0.03 \mathrm{Myr}$ ), and this value was left fixed thereafter. Here we continue to use that value.

In addition, in eq. (3) $), M\left(n>n_{\mathrm{SF}}, t\right)$ is the mass of the material at densities above $n_{\mathrm{SF}}$, and given by

$$
M\left(n>n_{\mathrm{SF}}, t\right)=f M_{\mathrm{C}}(t)
$$

where $f$ is the mass fraction at densities above $n_{\mathrm{SF}}$, given by ${ }^{2}$

$$
f=\frac{1}{2}\left[1-\operatorname{erf}\left(\frac{s_{\mathrm{SF}}-\sigma_{s}^{2} / 2}{\sqrt{2} \sigma_{s}}\right)\right],
$$

\footnotetext{
${ }^{2}$ Note that this equation in Paper I contains a typographical error. The form written here is the correct expression.
} 
and $s_{\mathrm{SF}} \equiv \ln \left(\rho_{\mathrm{SF}} /\langle\rho\rangle\right)$ (see also Elmegreen 2002; Krumholz \& McKee 2005; Dib et al. 2011).

As mentioned above, in contrast with the models by Krumholz \& McKee (2005), Padoan \& Nordlund (2011), and Hennebelle \& Chabrier (2011), which considered stationary clouds, here we assume that the cloud is collapsing, keeping in mind that it is a sheet-like object, and so its collapse proceeds more slowly than that of a three-dimensional object of the same volume density (Burkert \& Hartmann 2004; Toalá et al. 2012; Pon et al. 2012). In the model, we numerically solve the free-fall motion of the sheet-like cloud.

As a consequence of its collapse, the mean density of the cloud increases with time, causing the density PDF to systematically shift towards higher densities. Thus, in our model (based on the notion of hierarchical gravitational collapse; Vázquez-Semadeni et al. 2009), the global collapse of the cloud is represented by the fact that the mean density of a cloud or clump increases over time, while the local collapses of the densest regions are represented by the calculation of the instantaneous SFR, performed by considering the mass above $n_{\mathrm{SF}}$ and dividing it by the free-fall time at this density. This treatment implies that the SFR in the model is an increasing function of time, since the area under the PDF at densities higher than $n_{\mathrm{SF}}$ increases as the mean density increases. The total mass in stars at time $t$ in the model is thus given by

$$
M_{*}(t)=\int_{0}^{t} \operatorname{SFR}\left(t^{\prime}\right) d t^{\prime}
$$

From this stellar mass, the number of massive stars (with a representative mass of $17 \mathrm{M}_{\odot}$ ) is computed using a standard IMF (Kroupa 2001), with lower and upper mass limits of 0.01 and $60 \mathrm{M}_{\odot}$, respectively. In turn, this allows us to compute the mass evaporation rate, $\dot{M}_{\text {I }}(t)$, by these stars using the prescription from Franco et al. (1994) for the evaporation rate induced by a single massive star of age $\hat{t}$ located near the cloud edge, given by

$$
\dot{M}_{\mathrm{I}}(\hat{t}) \simeq 2 \pi R_{\mathrm{S}, 0}^{2} m_{\mathrm{p}} c_{\mathrm{s}, \mathrm{I}}\langle n\rangle\left(1+\frac{5 c_{\mathrm{s}, \mathrm{I}} \hat{t}}{2 R_{\mathrm{S}, 0}}\right)^{1 / 5}
$$


where $c_{\mathrm{S}, \mathrm{I}}$ is the sound speed in the ionized gas $\left(=12.8 \mathrm{~km} \mathrm{~s}^{-1}\right),\langle n\rangle$ is the mean number density of the cloud, $m_{\mathrm{p}}$ is the proton mass, and $R_{\mathrm{S}, 0}$ is the initial Strömgren radius in a medium of density $\langle n\rangle$. To calculate this radius, as in Franco et al. (1994), we additionally assume a recombination coefficient for the ionized gas $\alpha_{\mathrm{B}}=2.6 \times 10^{-13} \mathrm{~cm}^{-3} \mathrm{~s}^{-1}$ and a representative value of the UV Lyman-continuum photon flux $S_{*}=2 \times 10^{48} \mathrm{~s}^{-1}$, corresponding to our generic massive star. Finally, to get the total ionized mass we integrate eq. (7) over the lifetime of each massive star formed and add the contributions from all active massive stars.
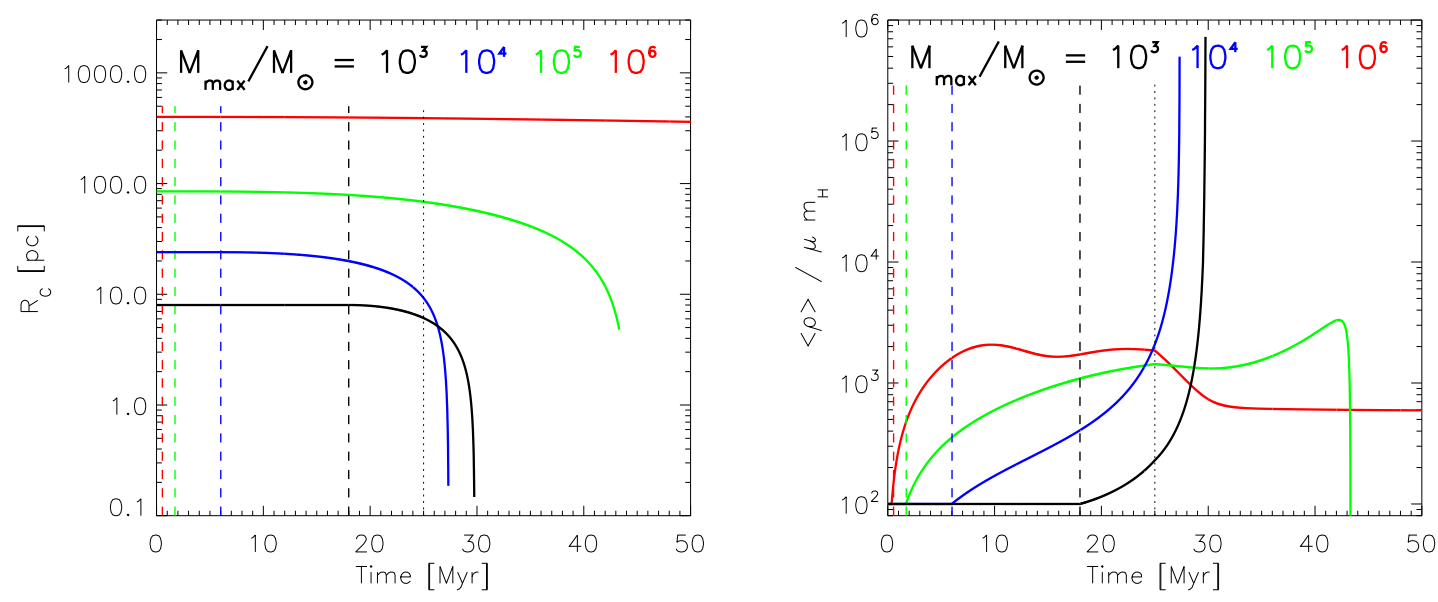

Fig. 1. - Left: Time evolution of the radius for clouds with $M_{\max }=10^{3}, 10^{4}, 10^{5}$, and $10^{6} \mathrm{M}_{\odot}$ (black, blue, green and red lines respectively). The vertical dashed lines represent the time at which the cloud reaches its Jeans mass and begins contracting, whereas the vertical thin dotted black line is the time at which the accretion stops (at $t=25 \mathrm{Myr}$ ). Right: Time evolution of the density. The line colors have the same meaning as in the left panel.

With the above ingredients, the instantaneous mass of the cloud is the result of the competition between addition of fresh gas by accretion, the consumption by star formation, and the evaporation from the massive stars. Thus, the cloud mass evolves according to

$$
M_{\mathrm{C}}(t)=\int_{0}^{t} \dot{M}_{\mathrm{inf}}\left(t^{\prime}\right) \mathrm{d} t^{\prime}-M_{*}(t)-M_{\mathrm{I}}(t) .
$$


We numerically integrate eq. (8), together with eqs. (6) and (7), to obtain the temporal evolution of a specific cloud, until it is finally dispersed.

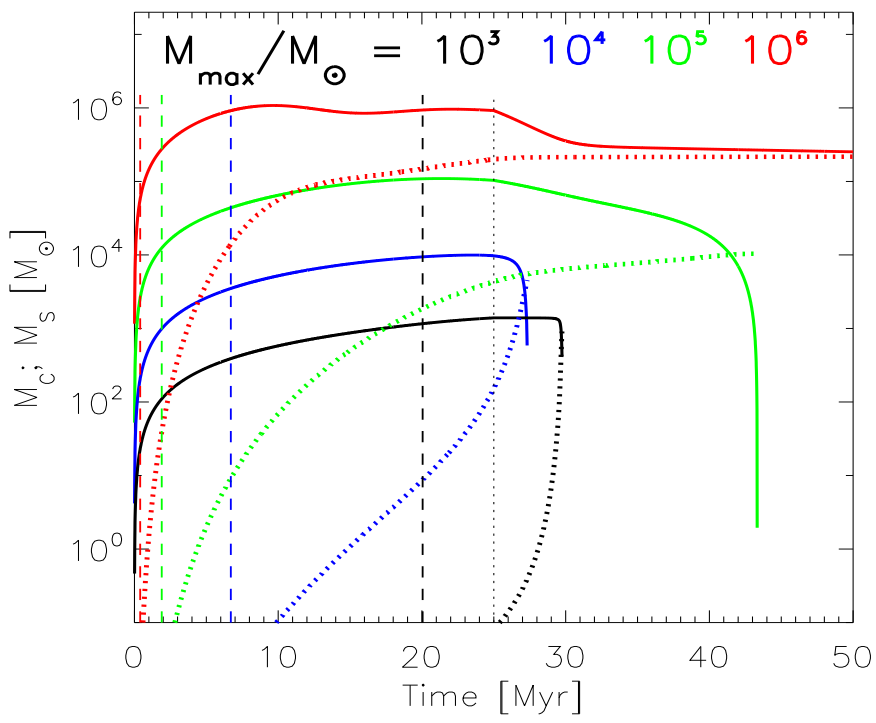

Fig. 2.- Time evolution of the dense gas mas (solid lines; eq. [8]), and mass in stars (dotted lines) for clouds with $M_{\max }=10^{3}, 10^{4}, 10^{5}$, and $10^{6} \mathrm{M}_{\odot}$ (black, blue, green and red lines respectively). The vertical dashed lines represent the time, $t_{\mathrm{SF}}$, at which the cloud starts to form stars, whereas the vertical thin dotted black line is the time at which the accretion stops (at $t=25 \mathrm{Myr}$ ).

As emphasized in Paper I, the main controlling parameter of this model is the total mass involved in the process, which, for fixed values of $\rho_{\mathrm{WNM}}$ and $v_{\text {inf }}$ is controlled by the cylinder radius $R_{\text {inf }}$. In what follows, we thus choose the required value of $R_{\text {inf }}$ to obtain the reported maximum cloud mass, which is the maximum mass reached by the cold, dense gas during the model's evolution, and labeled $M_{\max }$. Since the numerical integration of the model takes only a few seconds on a desktop computer, it allows us to sweep parameter space using hundreds of models of different masses, a task that cannot be undertaken with full numerical simulations. 
Figure 1 shows the evolution of the cloud radius and mean density for representative clouds with $M_{\max }=10^{3}, 10^{4}, 10^{5}$, and $10^{6} \mathrm{M}_{\odot}$. A number of features are worth noticing. First, from the left panel of Fig. 1, it is seen that the radius of a cloud remains essentially constant over more than 10 Myr of evolution, during which the cloud is accreting mass, until it reaches its Jeans mass. Afterwards, the cloud's radius begins to decrease at an accelerated pace, with its mean density increasing, as shown by the right panel of Fig. 1. It is noteworthy that this evolution implies that the material constituting an initially medium-sized cloud, of size $\sim 10$ pc and mass a few thousand $\mathrm{M}_{\odot}$, such as Perseus or Ophiuchus, should evolve into a massive-star-forming clump, of density $\sim 10^{5}-10^{6} \mathrm{~cm}^{-3}$ and sizes $\lesssim 1$ pc, such as the massive clumps studied by Wu et al. (2010), as shown in Fig. 7 of Paper I. It must be borne in mind, however, that, by the time the cloud has contracted to a massive clump, it is embedded in the envelope that has been added to the cloud's surroundings by the WNM inflows.

Figure 2, in turn, shows the evolution of the dense gas mass and the stellar mass for these models. It is seen that in all but the most massive model (the one with $\left.M_{\max }=10^{6} \mathrm{M}_{\odot}\right)$, the cloud mass increases until the time when the stellar ionizing feedback begins to rapidly erode the dense gas mass, causing it to decrease again. The exception to this behavior is the model with $M_{\max }=10^{6} \mathrm{M}_{\odot}$, for which the dense gas mass stops increasing at $t \sim 10 \mathrm{Myr}$. This is due to the fact that its mass is so large that the mass fraction at high density in this cloud allows for the formation of massive stars and the corresponding erosion even before the cloud has had time to contract significantly 3 .

\footnotetext{
${ }^{3}$ Recall that in the model, the clouds are assumed to have sheet-like geometry, and that their collapse is given by the expression corresponding to such geometry (Burkert \& Hartmann 2004), which is slower than the collapse for a spherical geometry (Toalá et al. 2012; Pon et al. 2012).
} 
In the next section we now discuss the evolution of the SFR and the SFE for the models as parameterized by their mass.

\section{Model Predictions}

\subsection{Mass Dependence of the Star Formation Rate}

In this and the following sections, we consider a collection of models of various masses, and focus on the variation of the maximum and time-averaged values of the SFR and the SFE (cf. Sec. 3.2) as a function of $M_{\max }$. The time averages we consider cover the time span between the formation of $0.01 \mathrm{M}_{\odot}$ (the lower limit in the IMF considered) of stellar products and the destruction of the cloud (see Fig. 2). To simplify the discussion, we will refer to "low-mass clouds" as those with $M_{\max } \lesssim 10^{4} \mathrm{M}_{\odot}$; to "intermediate-mass" clouds as those with maximum masses in the range of $10^{4}-10^{5} \mathrm{M}_{\odot}$, and to "massive clouds" as those with $M_{\max } \gtrsim 10^{5} \mathrm{M}_{\odot}$.

It is important to note that, so far, we have referred to our models simply as "clouds". However, in this section, in which we try to predict characteristic values of the SFR and SFE (characterized by their time averages) in molecular clouds of different masses, it is important to define the time interval during which the clouds can be considered as "molecular", so that the time averages are computed over this interval. Unfortunately, in

our one-zone model without chemistry, there is no direct way to determine this time. Thus, we instead take the beginning of the averaging interval as the time at which the clouds begin to form stars, $t_{\mathrm{SF}}$. We have verified that this is a reasonable proxy for determining when the clouds begin to be sufficiently molecular by using the density PDF to compute the mass fraction at densities $n>10^{3} \mathrm{~cm}^{-3}$ — which can be reasonably assumed to be already in molecular form - at the time SF starts, finding that in all models this mass fraction is 
$\geq 0.2$. Thus, hereinafter we will refer to clouds after $t_{\mathrm{SF}}$ as "star-forming molecular clouds" (SF-MCs), and to clouds in previous stages as "precursor" clouds.

In the left panel of Fig. 3, we show the evolution of the SFR for clouds of maximum masses $M_{\max }=10^{3}, 10^{4}, 10^{5}$, and $10^{6} \mathrm{M}_{\odot}$. We observe that low-mass SF-MCs have a very low SFR over most of their evolution, and end their evolution with a short SF burst. This can be understood because, due to their low mass, these clouds can only reach large SFRs when a substantial fraction of their mass is involved in SF. This can only occur when their mean density has become comparable to $n_{\mathrm{SF}}$, as shown in the right panel of Figure 1. At this point, these clouds are quickly destroyed by the first massive stars. This also means that the consumption of the gas in these clouds is mostly due to SF, rather than to evaporation by feedback from the massive stars.

On the other hand, intermediate- and high-mass SF-MCs increase their SFR for the first $\sim 10 \mathrm{Myr}$, and then reach a plateau, remaining there for roughly 10-20 Myr more. This is because, due to their higher masses, they can have larger SFRs since earlier times (even a small fraction of their mass corresponds to a large enough mass involved in active SF). This implies that massive SF starts earlier in these clouds. Nevertheless, due to their larger masses and accretion rates, they are not completely destroyed, although these clouds do lose some of their mass when the first massive stars appear. Moreover, this partial mass dispersal causes a decrease in the SFR, and therefore the cloud evolution reaches an approximately stationary SFR for a significant part of their lifetimes. Thus, in these clouds, a larger fraction of the dense gas consumption is due to evaporation, compared to the low-mass clouds. Note, however, that accretion plays a fundamental role in this behaviour since, in experiments where we have cut the accretion shortly after the onset of SF, the massive stars quickly destroy the clouds.

In the right panel of Figure 3, we show the maximum and the time-averaged values of 
the SFR as a function of $M_{\max }$. Note that each point in this figure corresponds to a full integration of an individual model. We note that, for low-mass SF-MCs, the maximum SFR is much larger than its average values, because of the short but intense SF burst that characterizes the end of the evolution of these clouds. Instead, for more massive SF-MCs, the peak and the average SFR are similar, due to the prolonged epoch of roughly constant $\mathrm{SF}$ that occurs in these clouds. As a reasonable approximation, the time-averaged SFR can
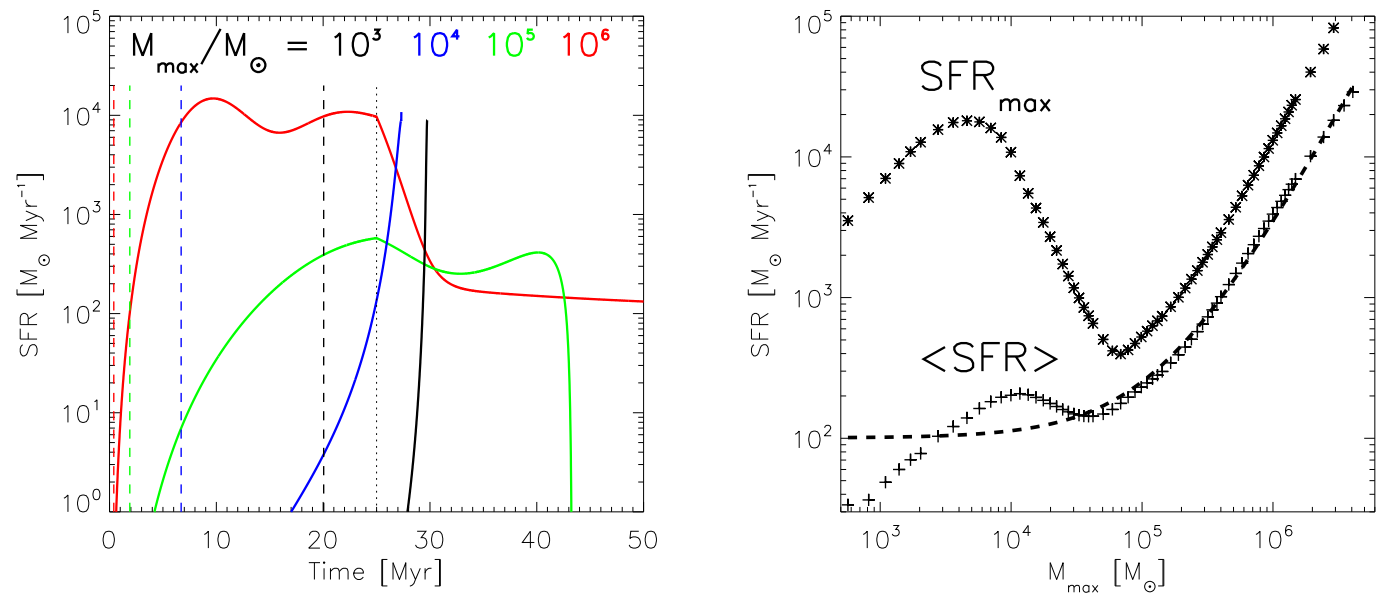

Fig. 3. - Left: Time evolution of the SFR for clouds with $M_{\max }=10^{3}, 10^{4}, 10^{5}$, and $10^{6} \mathrm{M}_{\odot}$ (black, blue, green and red lines respectively). The vertical dashed lines represent the time, $t_{\mathrm{SF}}$, at which the cloud starts to form stars, whereas the verical thin dotted black line is the time at which the accretion stops (at $t=25 \mathrm{Myr}$ ). Right: Maximum and time-averaged SFR asterisks and plus symbols respectively) as a function of the maximum mass achieved by each model cloud. The averaging is performed over the period during which the clouds form stars.

be fit by a power law of the cloud mass, given by

$$
\langle\mathrm{SFR}\rangle \approx 100\left(1+\frac{M_{\max }}{1.4 \times 10^{5} \mathrm{M}_{\odot}}\right)^{1.68} \mathrm{M}_{\odot} \mathrm{Myr}^{-1}
$$

which is shown as the dashed line in the right panel of Fig. 3, 


\subsection{Mass Dependence of the Star Formation Efficiency}

We now turn to the mass dependence of the SFE. As in Paper I, we define the instantaneous SFE as

$$
\operatorname{SFE}(\mathrm{t})=\frac{M_{*}(t)}{M_{\mathrm{C}}(t)+M_{*}(t)+M_{\mathrm{I}}(t)},
$$

where all the quantities are time-dependent. The left panel of Fig. 4 shows the time

evolution of the SFE for models with $M_{\max }=10^{3}, 10^{4}, 10^{5}$, and $10^{6} \mathrm{M}_{\odot}$. From this figure, we see that in the low-mass SF-MCs, the final star formation burst (see right panel in Fig. 3) produces large final efficiencies $(\lesssim 60 \%)$, although this is not in contradiction with observations, as it is not possible to observationally determine the SFE of a cloud/cluster system after the gas has been dispersed. On the other hand, for the more massive SF-MCs, the SFE reaches a saturated value of $\sim 6 \%$. The SFE can saturate due to the interplay between the gas evaporation by the feedback and the accretion of fresh gas, so that the masses of the cloud and of the stellar component increase simultaneously, keeping the instantaneous SFE approximately constant.

The right panel of Fig. 4 shows the final and time-averaged efficiencies for the SF-MCs as a function of their masses. As in the right panel of Fig. 3, each point in this plot represents the full temporal integration of one model with a given radius $R_{\text {inf }}$, which reaches the maximum dense gas mass indicated by its horizontal coordinate. From this figure, we see that, although the final instantaneous SFEs of the low mass clouds are much higher than those of the high-mass ones, the time-averaged values of the SFE increase monotonically with $M_{\max }$. The time-averaged SFE should be representative of the result of observing a $\mathrm{MC}$ ensemble of random ages, and thus represent the average value of the SFE observed for MCs of the indicated mass. We see that $\langle\mathrm{SFE}\rangle$ is well fit by a power law of the form

$$
\langle\mathrm{SFE}\rangle \approx 0.03\left(\frac{M_{\max }}{2.5 \times 10^{5} \mathrm{M}_{\odot}}\right)^{0.33},
$$

so that we obtain time-averaged SFEs in the range of $0.5-6 \%$ for the range of maximum 
cloud masses shown in Fig. 4, consistent with observational determinations for GMCs in general (see, e.g., the compilation by Federrath \& Klessen 2013). Thus, our model predicts that the time-averaged SFE should increase with the cloud mass, albeit slowly, with a scatter that might correspond not only to observational errors, but also to the variation of the SFE over the evolution of the clouds.
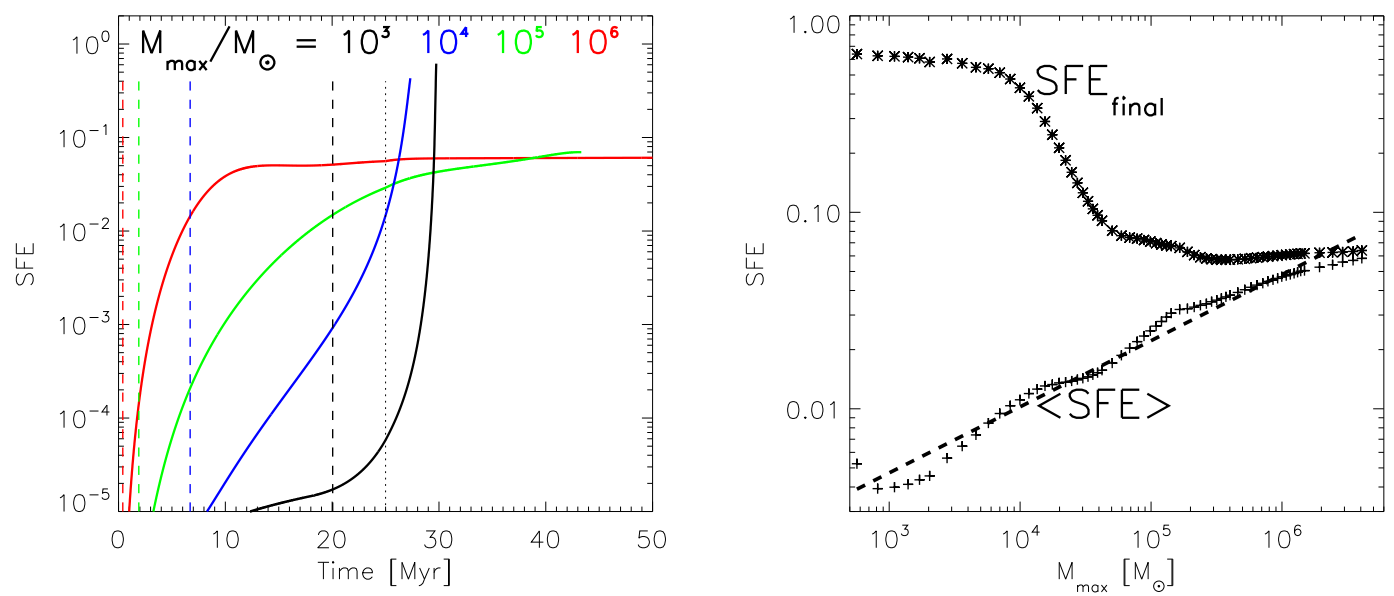

Fig. 4. - Left: Time evolution of the SFE for clouds with $M_{\max }=10^{3}, 10^{4}, 10^{5}$, and $10^{6} \mathrm{M}_{\odot}$. The symbolism is the same as in Fig. 3. Right: Maximum and mean SFEs as a function of the maximum cloud mass.

The exponent in eq. (11) is close to the value predicted analytically by Fall, Krumholz \& Matzner (2010) of 0.25 for the case of feedback dominated by ionization heating. The difference may be due to the fact that those authors considered stationary energy (or momentum, for the case of momentum-dominated feedback) balance, while here we take the additional step of considering the time evolution of the feedback, and/or to the different assumed geometries (flat in our case). 


\subsection{Star formation rate-dense gas mass correlation}

In Paper I we showed that the evolution of our model clouds with maximum dense gas masses $M_{\max } \sim 2000 \mathrm{M}_{\odot}$, when plotted in the Kennicutt-Schmidt (KS) diagram, took them from the locus of clouds forming low-mass stars, such as Perseus, Lupus, Serpens, and

Ophiuchus (Evans et al. 2009), to that of clouds forming massive stars, such as the Orion A cloud and the clumps investigated by Heiderman et al. (2010). We now investigate whether, collectively, our clouds conform to observed star formation correlations found for averages over large volumes. This is important because those correlations are often interpreted as the result of a sustained low value of the SFE due to global turbulent support of the clouds (e.g., Krumholz et al. 2009, 2012), while our model clouds are in global collapse, and their SFR and SFE are not constant, but rather, time-dependent. In this case, one can ask whether the time-averaged (over their star-forming epoch) SFR and SFE of our model clouds are consistent with the observed SF correlations.

One important such correlation is the one found by Gao \& Solomon (2004, herafter GS04) who, in a sample of luminous and ultraluminous infrared galaxies, as well as of normal spirals, found a linear relationship between the IR luminosity (a tracer of the SFR) and the HCN luminosity (a tracer of the dense $\left[n \geq n_{\text {dens }}=3 \times 10^{4} \mathrm{~cm}^{-3}\right]$ gas mass), implying that

$$
\mathrm{SFR} \approx 180\left(\frac{M_{\text {dens }}}{10^{4} \mathrm{M}_{\odot}}\right) \mathrm{M}_{\odot} \mathrm{Myr}^{-1}
$$

which is a linear relationship between the SFR and $M_{\text {dens }}$, the mass at density $\geq n_{\text {dens }}$ (Fig. 51). On MC scales, Lada et al. (2010, hereafter LLA10) found a similar linear relationship $\left(\mathrm{SFR} \propto M_{\text {dens }}\right)$ for a sample of nearby MCs (see Fig. 5), measuring the gas mass at densities above $n \geq n_{\text {dens }}=10^{4} \mathrm{~cm}^{-3}$ from extinction maps, and estimating the SFR by counting young stellar objects (YSOs) and dividing by a typical age spread, $\Delta t \sim 2$ Myr. These authors also found that the SFEs of their cloud sample fall in the range of $0.1-4 \%$. 
We wish to compare our model's predictions to these results. To do so, at the individual cloud level, we attempt to replicate the procedure of LLA10. Note that, according to our model, both the SFR and the SFE of a cloud increase over time, and thus the range of efficiencies observed in LLA10's sample is interpreted as a spread in evolutionary stages. In turn, this implies that the corresponding SFRs should also correspond to a range of evolutionary stages. Thus, for each model cloud, we should consider the range of SFRs that it may have over this range of evolutionary stages for comparison with the observations. However, we have the problem that the evolutionary stage of the clouds considered by LLA10 is not known. To circumvent this problem, we make use of the fact that our model predicts both the SFR and the SFE of the clouds as a function of time. These quantities are different from each other, because the SFR is a truly instantaneous quantity, while the SFE involves the integral of both the stellar and gaseous mass accretion rates (cf. eq. [10]). Thus, we can use the instantaneous SFE of a cloud as a proxy for its evolutionary stage, and then compute the corresponding SFR predicted by the model at that stage, to compare with the observationally-inferred SFRs.

Note, in addition, that the SFR estimates by LLA10 are not strictly instantaneous values, but rather, averages over the age spread $\Delta t$. We thus also estimate the SFR not through the instantaneous SFR predicted by the model, but as the number of stars of age $<2$ Myr, divided by this time interval, and compute this estimate at two different times, one, labeled $t_{1}$, when the clouds' SFE is $0.1 \%$ and the other, labeled $t_{2}$, when the clouds' SFE is $4 \%$, consistent with the range of SFEs exhibited by the LLA10 sample. Thus, for each model cloud we report a range of SFRs. Similarly, the instantaneous mass of the model clouds varies between these two times, and therefore we also report a range of masses for each model cloud. We do this for model clouds with maximum masses in the same mass range - recall the model clouds are labeled by the maximum mass they reach during their evolution - as the clouds in their sample. 


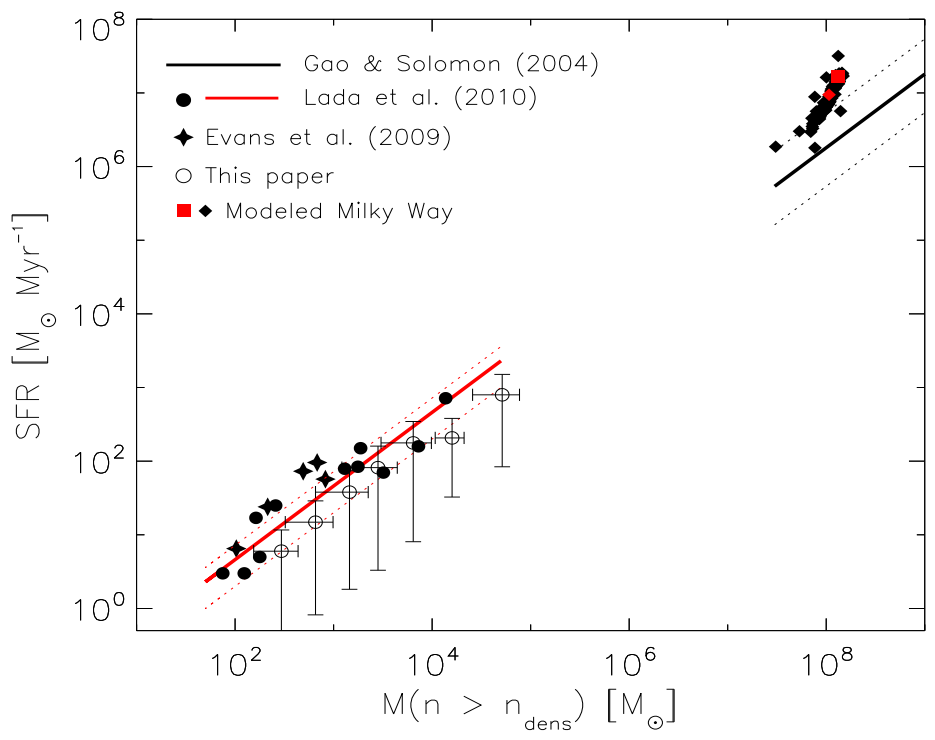

Fig. 5.- SFR as a function of dense gas mass $\left(M_{\text {dens }}=M\left(n \geq n_{\text {dens }}\right)\right.$, with $n_{\text {dens }}=$ $10^{4} \mathrm{~cm}^{-3}$ ) for low- to intermediate-mass model clouds (open circles with error bars; see text). In the lower-left corner, corresponding to individual cloud masses, the filled stars correspond to data from Evans et al. (2009), while the filled circles correspond to the cloud sample studied by LLA10, with the red solid line denoting the mean fit reported by those authors, and its dispersion represented by the red dotted-lines. In the upper-right corner, corresponding to galactic masses, the solid black line shows the scaling found by GS04 (eq. 12, with $n_{\text {dens }}=3 \times 10^{4} \mathrm{~cm}^{-3}$ ), the black dotted-lines showing the scatter of their observational sample. The filled red square shows the position of the modeled Milky Way given by eq. (14). Finally, the filled black dimonds represent Monte Carlo realizations of cloud ensembles taken at random evolutionary stages (in the SF-MC stage), with the red filled diamond giving the average value of these experiments.

In Fig. 5 we show, in the lower-left corner, the range of SFRs computed as described above versus the range of dense gas mass (i.e., at densities $n>n_{\text {dens }}=10^{4} \mathrm{~cm}^{-3}$ ) for our model clouds (open circles with error bars) and for the cloud sample studied by LLA10 
(filled black circles) and by Evans et al. (2009) (filled black stars). 4 The ranges of SFR and $M_{\text {dens }}$ between $t_{1}$ and $t_{2}$ for the model clouds are indicated by the error bars, while the open circles are the mean of these quantities between these two times. It is seen that the slope of the ensemble of models is nearly unity, similarly to the fit by LLA10, and that the locus of the means falls within the scatter reported by those authors.

As a further test of the model, we can estimate the total Galactic SFR it predicts by convolving the time-averaged SFR for each cloud mass, $\langle\mathrm{SFR}\rangle(M)$, with a suitable cloud mass spectrum. This can be then compared with the average relation derived by GS04 for external galaxies. by

$$
d N_{\mathrm{C}}=N_{0}\left(\frac{M_{\mathrm{U}}}{M}\right)^{\alpha} d(\ln M)
$$

where $d N_{\mathrm{C}}$ is the number of MCs with masses in the range $M$ to $M+\mathrm{d} M, N_{0}=63$, $\alpha=0.6$ and $M_{\mathrm{U}}=10^{6} \mathrm{M}_{\odot}$ is the maximum assumed mass of GMCs in the Galaxy (see also McKee \& Ostriker 2007). The total Galactic SFR is then given by

$$
\mathrm{SFR}_{\text {tot }}=\int_{0}^{M_{\mathrm{U}}}\langle\mathrm{SFR}\rangle(M) d N_{\mathrm{C}}
$$

This exercise gives a global Galactic SFR of $14 \mathrm{M}_{\odot} \mathrm{yr}^{-1}$, within a factor of 5 from recent observational estimates (e.g., Chomiuk \& Povich 2011). Also, we can compute the total

\footnotetext{
${ }^{4}$ LLA10 report the dense gas mass, and we take the number directly from them. However, Evans et al. (2009) only report total masses, and hence we estimate $M_{\text {dens }}$ for their cloud sample using the same procedure as for our model clouds; that is, we assume a lognormal PDF centered at the mean density of the clouds reported by those authors, and with width corresponding to a Mach number of 3.
} 
dense mass above $n_{\text {dens }}$ as

$$
M_{\mathrm{tot}}\left(n>n_{\mathrm{dens}}\right)=\int_{0}^{M_{\mathrm{U}}}\left\langle\mathrm{M}\left(\mathrm{n}>\mathrm{n}_{\mathrm{dens}}\right)\right\rangle(M) d N_{\mathrm{C}}
$$

where $M\left(n>n_{\text {dens }}\right)$ is given by eqs. (41) and (5), replacing $n_{\mathrm{SF}}$ by $n_{\text {dens }}$. We find $M_{\text {tot }}\left(n>n_{\text {dens }}\right)=1.3 \times 10^{8} \mathrm{M}_{\odot}$. The red filled square near the upper-right corner of Fig. 5 shows the resulting "model galaxy", based on our model's predicted $\langle\mathrm{SFR}\rangle$, which is seen to be larger than the mean scaling by GS04 by a factor $\sim 5$. The discrepancy is probably due to the strong SF bursts predicted by our model for low-mass clouds, in which $\sim 40 \%$ of the total SFR takes place, according to the mass spectrum.

A perhaps more precise comparison is provided by a Monte Carlo integration, taking the SFR and the corresponding mass at random times for each SF-MC, and integrating again according to the mass spectrum. In the upper-right corner of Fig. 5 (filled black diamonds) we also show a hundred of these experiments, obtaining average values of $9 \mathrm{M}_{\odot} \mathrm{yr}^{-1}$ and $M_{\text {dens }}=1.1 \times 10^{8} \mathrm{M}_{\odot}$ for the total SFR and dense mass gas (filled red diamond), respectively. Roughly a third of the points generated in this way are seen to fall within the uncertainties of the GS04 relation. Nevertheless, the set of points always falls above the mean GS04 relation, and so the model does seem to overestimate the Galactic SFR by a factor of $3-5$. We discuss this further in Sec. 4.2.

\section{Discussion and limitations}

\subsection{Implications and insights}

One important prediction of our model is that GMCs evolve in such a way that a moderate-size MC eventually becomes a dense, massive-star-forming clump, over the course of $\sim 10$ Myr. We now discuss how this process fits into our established knowledge about MCs. 


\subsubsection{Large- and small-scale collapse}

The first point to emphasize is that the model is designed to account for both the collapse of small-scale clumps and cores and the collapse of the cloud as a whole. This

is in line with the notion advanced by Vázquez-Semadeni et al. (2009) that gravitational collapse in MCs is hierarchical so that small-scale, local collapses occur within the environment of a cloud that is also undergoing collapse as a whole (a large-scale collapse). The small-scale collapse is described in the "standard" way (e.g., Krumholz \& McKee 2005; Hennebelle \& Chabrier 2011; Padoan \& Nordlund 2011), by assuming that the cloud contains a distribution of density fluctuations, the densest of which are undergoing instantaneous collapse, and therefore being responsible for the instantaneous SFR of the cloud. This amounts for what is normally referred to as the fragmentation of the cloud.

The large-scale collapse, on the other hand, is accounted for by directly computing the contraction rate of the whole cloud based on its average density and corresponding free-fall time. This contrasts with the models cited above, which assume roughly stationary conditions in the clouds, and therefore cannot account for any evolutionary features of the clouds. In our model, the whole evolutionary nature of the process derives from the fact that the cloud is contracting as a whole.

\subsubsection{Velocity gradients: rotation or infall?}

A widespread notion is that MCs rotate, and that such rotation would prevent their contraction to clump scales. Indeed, velocity gradients are ubiquitously observed in MCs \begin{tabular}{ll|l|l|l|}
\hline (e.g., Pound \& Goodman 1997; & Rosolowskv et al. 2003; Brunt 2003; Brunt et al. 2009) as
\end{tabular} well as in dense cores within them (e.g., Goodman et al. 1993; Kirk et al. 2010, see also the review by Belloche, 2013). However, as stated above, some form of contraction must 
occur in order to form a massive, dense clump.

Although the velocity gradients are almost always interpreted as rotation, it is important to remark that there is no a priori reason to do this, and in fact, Brunt (2003) points out that the Principal Component Analysis of the velocity structure in MCs is inconsistent with the signature of rotation in model clouds. On the other hand, Vázquez-Semadeni et al. (2008) showed that overdense regions in simulations of driven isothermal turbulence exhibit on average a negative velocity divergence (i.e., a velocity convergence) whose magnitude is within the range of velocity gradients reported by Goodman et al. (1993) in cores of similar sizes, suggesting again that a significant component of the observed gradients may actually consist of inflow motions, rather than rotation. Similar conclusions were obtained by Csengeri et al. (2011) for massive dense cores in Cygnus-X, where they concluded that infall as well as rotation may be present.

Finally, it should also be noticed that, in general, these infalling motions are not expected to be spherically symmetrical, as MCs are observed to consist mostly of filamentary structures (e.g., Myers 2009; André et al. 2010; Molinari et al. 2010; Kirk et al. 2013), and therefore the classical infall signature (e.g., Evans 1999, sec. 4.7) should not be expected in molecular line observations of objects at these scales, so the failure to detect them does not rule out the possibility that the velocity gradients observed across clouds correspond in fact to collapsing motions.

\subsubsection{Delayed and extended star-formation activity}

Another prediction from the model is that the evolution of the clouds includes a period of time (see left panel of Fig. 3) with no significant SF (i.e., as precursor clouds) and, once star formation starts, they are expected to spend several more Myr at low SFRs. 
Interestingly, the low-mass clouds spend longer times in these states than the high-mass ones. For example, it can be seen in Fig. 3 that a cloud with $M_{\max }=10^{3} \mathrm{M}_{\odot}$ takes $\sim 20 \mathrm{Myr}$ to start forming stars, and after that, it spends $\sim 8$ Myr with very low SFR. This is because the low-mass clouds need to contract by a large factor to reach a large enough mean density that the mass above the threshold density $n_{\mathrm{SF}}$ causes a significant SFR. Instead, the high-mass clouds begin to do so at earlier times, when their mean density is still relatively low, because even a small fraction of their mass above $n_{\mathrm{SF}}$ involves sufficient mass for the SFR to already be significant.

However, this prediction might appear contradictory with the notion that MCs form stars rapidly after their formation (Hartmann, Ballesteros-Paredes \& Bergin 2001). What must be borne in mind here is that the model follows the evolution of the clouds from their earliest, cold-atomic stage, which is effectively the precursor of a GMC (Vázquez-Semadeni et al. 2006). Clouds formed by colliding WNM streams are expected to build up their molecular content over relatively long timescales (e.g., Franco \& Cox 1986; Hartmann, Ballesteros-Paredes \& Bergin 2001; Heitsch \& Hartmann 2008; Micic et al. 2013) and should only become mostly molecular by the time when they have become strongly gravitationally bound. In the context of our model, then, the zero- or low-SFR epochs correspond to times when the cloud is still atomic-dominated, consisting of a collection of moderate-mass, slowly-star-forming molecular clumps, immersed in an atomic substrate. The high-SFR stages occur when the cloud is already in a mostly molecular state, in agreement with the notion that MCs form stars rapidly, within a few Myr from their formation. The subtle additional consideration is that the clouds have much longer time spans, but during most of that time, they are mostly non-molecular, and they are forming stars at very low rates. We estimate the molecular fraction of the clouds' mass as a function of time for the sample clouds of masses $10^{3}$ to $10^{6} \mathrm{M}_{\odot}$, and in all cases, by the time SF starts, $20 \%$ or more of the mass is "molecular" (gas with number density greater 
than $\left.10^{3} \mathrm{~cm}^{-3}\right)$.

We emphasize that this result is consistent with the fact that embedded clusters generally contain a small fraction of older stars, although the majority of their stars is young (Palla \& Stahler 1999, 2000). Indeed, in Paper I we showed that the model correctly recovers the typical age distributions found in those clusters. Also in this regard, it should be noted that the above discussion implies that the gravitational contraction is likely to start in the mostly-atomic stage. This is consistent with numerical simulations of cloud formation that also follow the atomic and molecular fractions, which suggest that molecules actually form during the gravitational contraction (Heitsch \& Hartmann 2008), and with the result by Glover \& Clark (2012) that molecules are in fact not necessary for producing the cooling needed for gravitational collapse.

Finally, an important point is worth clarifying. The prediction that massive GMCs, with $10^{5}-10^{6} \mathrm{M}_{\odot}$, have extended periods of star formation lasting 20 Myr or more may seem to be in conflict with observations of nearby clouds like Orion and "dispersed" regions, such as Sco-Cen, as it is generally accepted that cloud dispersal by the recently-formed stars occurs rapidly, within a few Myr. However, this quick dispersal refers essentially to the immediate gaseous environs of a newly formed cluster only, as it is also known that SF occurs only over a small fraction of a cloud's volume (e.g., Kirk et al. 2006), while the destruction of a large GMC may easily take over 10 Myr. For example, in the Sco-Cen region, the three main subgroups, Upper Scorpius, Upper Centaurus-Lupus, and Lower centaurus-Crux, have ages that differ by more than $10 \mathrm{Myr}$, and it has been suggested that the latest events might have been triggered by the earlier ones (e.g., Preibisch \& Mamajek 2008). Thus, SF in the parent GMC might have been going on for at least that amount of time, suggesting that the lifetime predicted by our model for massive clouds is reasonable. In the case of this complex, however, the dispersal has probably been completed already, as 
the clusters there are already devoid of gas. But the SF episode must have lasted at least the length of time spanned by the age difference between the clusters.

On the other hand, Kawamura et al. (2009) have suggested that GMCs with masses $\sim 10^{5}-10^{6} \mathrm{M}_{\odot}$ in the LMC may have lifetimes of $\sim 25 \mathrm{Myr}$, with three well-defined stages in terms of their SF activity. In Paper I we showed that our model, for clouds of those masses, matches within a factor of 2 the duration of the individual stages, while the left panel of Fig. 3 here shows that indeed this is the time span of the star-forming stages of such clouds.

Thus, we conclude that the cloud evolution predicted by the model is in very good agreement with many known properties and evolutionary features of MCs.

\subsection{Limitations}

Our SFR model is clearly an extreme idealization of the actual process occurring in MCs, as it only considers the effects of self-gravity and photoinonization on the evolution of the clouds. In particular, it neglects any support from magnetic fields, which are known to retard the gravitational collapse in comparison with the non-magnetic case (e.g.,

Ostriker et al. 1999), the momentum injection by the ionizing stellar feedback and by stellar outflows, and the additional feedback from supernovae and radiation pressure from the most massive stars. Since all of these processes tend to either counteract the collapse or to destroy the clouds more rapidly, it is clear that the SFR and SFE predicted by the model are upper limits to those in real clouds.

Nevertheless, it is all the more interesting that, within these important limitations, our model in general predicts values of the SFR and the SFE, as well as evolutionary features of the clouds (Paper I), that generally agree well with the corresponding observational measurements, with the largest deviations occurring when the time-averaged values of the 
SFR for all cloud masses are added to construct a Galaxy-wide SFR. Better agreement is obtained for the Galaxy-wide SFR when a set of Monte-Carlo realizations is considered, using values of the instantaneous SFR at random times for each cloud mass. This suggests that the final SFR burst predicted by the model for low-mass clouds may be overestimated, and indeed, the time-averaged SFR predicted for these clouds exhibits a bump at low-to-intermediate cloud masses (see Fig. 3). This suggests that, especially for these clouds, the effects of magnetic fields and outflows may be most important. Nevertheless, the general better-than-order-of-magnitude agreement of the model with the observations suggests that self-gravity and photoionizing radiation, the processes considered by the model, are among the dominant processes controlling the evolution of the clouds and their star formation activity, with the other processes providing second-order corrections only.

On the other hand, possibly the most questionable ingredient of our model is the assumption that the density PDF remains lognormal during the entire evolution of the clouds, an assumption that appears in conflict with the well-known result, from both observations and numerical simulations, that star-forming clouds develop a power-law high-density tail in their column density distributions (e.g., Kainulainen et al. 2009; Kritsuk et al. 2011; Ballesteros-Paredes et al. 2011; Girichidis et al. 2014). However, in Paper I we argued that turbulence alone produces a lognormal, which is the seed of subsequent gravitational collapse, and that the power-law tail is a result of this contraction. Thus, the mass in this regime perhaps should not be counted as a seed for subsequent collapse, since it is already undergoing collapse. In any case, to minimize the impact of this assumption, in Paper I we calibrated the value of the $n_{\mathrm{SF}}$ by matching the predictions of the model to the output of the self-consistent numerical simulations of Vázquez-Semadeni et al. (2010). The a-posteriori confirmation of this procedure is that, using the lognormal, the model was able to match a variety of observations. 
Another important point to recall is that the model assumes clouds with a flattened geometry, for which the collapse timescale is significantly longer than for a spherical geometry, typically by factors of half to one order of magnitude, than the standard free-fall time (Toalá et al. 2012; Pon et al. 2012). However, this is probably a reasonable assumption, since most clouds are known to consist of flattened or filamentary structures (e.g., Bally et al. 1989). This suggests that another important factor determining the SFR is the non-spherical geometry of MCs.

\section{Summary and Conclusions}

In this paper, we have presented the predictions for the dependence of the time-averaged SFR and SFE on the mass of the parent cloud from our semi-analytical model for the evolution of these quantities in gravitationally collapsing clouds, introduced in Paper I. The model assumes that the cloud forms by the collision of two streams in the WNM, which induces a transition to the cold phase, forming a cold cloud that becomes turbulent due to various instabilities (Heitsch et al. 2005, 2006; Vázquez-Semadeni et al. 2006). Soon this turbulent cloud begins to undergo global gravitational collapse. The collapse is hierarchical, because the turbulence in the cloud produces density fluctuations that have shorter free-fall times than the cloud as a whole, and then form stars before the collapse of the largest scales is completed. The fraction of the cloud's mass involved in instantaneous SF is determined by assuming that the density PDF in the cloud is lognormal, and that only the mass above a certain critical density, $n_{\mathrm{SF}}$, is instantaneously forming stars. As the cloud collapses, its mean density increases, so that the PDF shifts to higher densities, causing the instantaneous SFR to systematically increase in time.

The total amount of gas converted into stars is distributed among stellar masses accorging to a standard IMF. The most massive stars produce ionizing radiation, which 
evaporates parts of the cloud through HII regions. While all this is happening, the cloud continues to accrete material from the converging flows. Thus, the evolution of the cloud is regulated by the competition between addition of fresh material by the accretion and the gas consumption by the SF itself, as well as by the evaporation by the ionizing radiation from the massive stars. The model neglects the magnetic field and any injection of momentum by the stellar feedback.

In Paper I, it was found that the total mass involved in the process is the main free parameter controlling the evolution of the clouds and their SFR. We quantify this parameter by the maximum dense gas mass reached by the clouds, $M_{\max }\left(n \geq 100 \mathrm{~cm}^{-3}\right)$. In the present contribution, we have considered the evolution, the final values, and the time averages over the star-forming epochs of the model clouds, of the SFR and SFE predicted by the model, as a function of the maximum dense gas mass attained by the model clouds. We have found that low-to-intermediate-mass model clouds $\left(M \lesssim 10^{4} \mathrm{M}_{\odot}\right)$ spend their early and intermediate evolutionary stages forming stars at low rates, while a strong star formation burst is produced during their final, dense stages (when they appear as a massive clump within a larger cloud), at which time massive stars appear and quickly destroy the cloud. Therefore, these clouds have a low time-averaged SFR $(\langle\operatorname{SFR}\rangle)$ but a high final SFR. Instead, in massive clouds $\left(M \gtrsim 10^{5} \mathrm{M}_{\odot}\right)$, massive stars appear from early in their evolution, and thus the ionizing feedback regulates the SFR almost from the beginning. This leads to a final SFR comparable with the average. We provided fits to the mass dependence of the time-averaged SFR and SFE, given by eqs. (9) and (11).

We then proceeded to investigate the relation between the SFR and the dense cloud mass, $M_{\text {dens }}$, for our model clouds, mimicking the procedure followed by LLA10. These authors estimated the SFR as the mass in YSOs (in our case, stars younger than 2 Myr) divided by this time interval. Since the clouds studied by LLA10 span a wide range in 
SFEs, we considered our model clouds in the time interval during which they span the same SFE range. We found that the mean values of the SFR and the clouds' mass during this time interval fall within the error bars of the mean relation reported by LLA10.

We also estimated the total Galactic SFR predicted by our model, by convolving the SFR (in average or taken it at a random time after the onset of star formation) for each cloud mass with the Galactic cloud mass spectrum by Williams \& McKee (1997). The average of a hundred of these random realiztions is within half an order of magnitude from the observed Galactic SFR, and from the scaling relation found by GS04 for the global SFR vs. galaxy mass of a sample of external galaxies.

With respect to the SFE, we find that for low-mass clouds, in the final star formation burst, the efficiency reaches final values $\sim 60 \%$, although these values are not in conflict with observations because they correspond to the stage when no dense gas mass is left around a cluster, at which point it is almost impossible to observationally know the initial amount of gas mass that went into the formation of the cluster. The time-averaged SFE, on the other hand, is $\sim 1 \%$, consistent with observational determinations performed on clusters still embedded in their parent clouds (e.g., Evans et al. 2009). For massive clouds, the SFE reaches values up to $6 \%$ (but with averages $\lesssim 5 \%$ ), consistent with the upper limits of SFE $(\sim 10 \%)$ determined in Federrath \& Klessen (2013).

We next discussed several implications of the model in the context of well established notions about MCs and their SF activity, arguing that, although some of the model predictions and implications may seem to be in conflict with those notions, upon closer examination no conflict exists, and instead the model offers a new insight about the evolution of MCs.

As pointed out in Sec. 4, the fact that our extremely idealized model, in which only self-gravity and ionizing feedback control the evolution of the SFR in the clouds, fits the 
observations typically within factors of a few, suggests that these may be the dominant controlling processes, with other processes, such as magnetic support and momentum injection from massive-star winds, probably providing mainly second-order adjustments.

We are thankful to the anonymous referee, whose constructive report helped us in improving the clarity and reach of the predictions and implications of our model. We also thankfully acknowledge partial finantial support by UNAM grant IN102912 to M.Z.-A and CONACYT grant 102488 to E.V.-S. 


\section{REFERENCES}

André P. et al., 2010, A\&A, 518, L102

Audit, E., \& Hennebelle, P. 2005, A\&A, 433, 1

Ballesteros-Paredes, J., Klessen, R. S., Mac Low, M.-M., \& Vazquez-Semadeni, E. 2007, Protostars and Planets V, 63

Ballesteros-Paredes, J., Vázquez-Semadeni, E., Gazol, A., et al. 2011, MNRAS, 416, 1436

Bally, J., Stark, A. A., Wilson, R. W., \& Langer, W. D. 1989, The Physics and Chemistry of Interstellar Molecular Clouds - mm and Sub-mm Observations in Astrophysics, 331,81

Banerjee, R., Vázquez-Semadeni, E., Hennebelle, P., \& Klessen, R. S. 2009, MNRAS, 398, 1082

Belloche, A. 2013, EAS Publications Series, 62, 25

Brunt, C. M. 2003, ApJ, 583, 280

Brunt, C. M., Heyer, M. H., \& Mac Low, M.-M. 2009, A\&A, 504, 883

Burkert, A., \& Hartmann, L. 2004, ApJ, 616, 288

Chomiuk, L., \& Povich, M. S. 2011, AJ, 142, 197

Csengeri, T., Bontemps, S., Schneider, N., Motte, F., \& Dib, S. 2011, A\&A, 527, A135

Dib, S., Piau, L., Mohanty, S., \& Braine, J. 2011, MNRAS submitted, arXiv:1102.3839

Elmegreen, B. G. 2002, ApJ, 577, 206

Elmegreen, B. G., \& Scalo, J. 2004, ARA\&A, 42, 211 
Evans, N. J., II 1999, ARA\&A, 37, 311

Evans, N. J., et al. 2009, ApJS, 181, 321

Fall, M., Krumholz, M. R., \& Matzner, C. D. 2010, ApJ, 710, L142

Federrath, C., Klessen, R. S., \& Schmidt, W. 2008, ApJ, 688, L79

Federrath C., Roman-Duval J., Klessen R. S., Schmidt W., \& Mac Low M., 2010, A\&A, $512, \mathrm{~A} 81$

Federrath, C., Klessen, R. S., 2012, ApJ, 761, 156

Federrath C., \& Klessen R. S., 2013, ApJ, 763, 51

Ferrière, K. M. 2001, Reviews of Modern Physics, 73, 1031

Franco, J., \& Cox, D. P. 1986, PASP, 98, 1076

Franco, J., Shore, S. N., \& Tenorio-Tagle, G. 1994, ApJ 436, 795

Galván-Madrid, R., Keto, E., Zhang, et al. 2009, ApJ, 706, 1036

Gao Y., \& Solomon P. M. 2004, ApJ, 606, 271

Girichidis, P., Konstandin, L., Whitworth, A. P., \& Klessen, R. S. 2014, ApJ, 781, 91

Glover, S. C. O., \& Clark, P. C. 2012, MNRAS, 421,9

Goldbaum, N. J., Krumholz, M. R., Matzner, C. D., \& McKee, C. F. 2011, ApJ, 738, 101

Goldreich, P., \& Kwan, J. 1974, ApJ 189, 441

Goodman, A. A., Benson, P. J., Fuller, G. A., \& Myers, P. C. 1993, ApJ, 406, 528

Gómez, G. C. \& Vázquez-Semadeni, E. 2013, Submitted to ApJ, arXiv:1308.6298 
Hartmann, L., Ballesteros-Paredes, J., \& Bergin, E. A. 2001, ApJ, 562, 852

Hartman, L., \& Burkert, A., 2007, ApJ, 654, 988.

Heiderman, A., Evans, N. J., II, Allen, L. E., Huard, T., \& Heyer, M. 2010, ApJ, 723, 1019

Heiles, C., \& Troland, T. H. 2003, ApJ, 586, 1067

Heitsch, F., Burkert, A., Hartmann, L., Slyz, A. D., \& Devriendt, J. E. G. 2005, ApJ, 633, L113

Heitsch, F., Slyz, A., Devriendt, J., Hartmann, L., \& Burkert, A. 2006, ApJ, 648, 1052

Heitsch, F., \& Hartmann, L. 2008, ApJ, 689, 290

Hennebelle, P., Banerjee, R., Vázquez-Semadeni, E., Klessen, R.S., \& Audit, E. 2008, A\&A, 486, L43

Hennebelle, P., \& Chabrier, G. 2011, ApJ, 743, L29

Kainulainen, J., Beuther, H., Henning, T., \& Plume, R. 2009, A\&A, 508, L35

Kawamura, A., et al. 2009, ApJS, 184, 1

Kirk, H., Johnstone, D., \& Di Francesco, J. 2006, ApJ, 646, 1009

Kirk, H., Pineda, J. E., Johnstone, D., \& Goodman, A. 2010, ApJ, 723, 457

Kirk, H., Myers, P. C., Bourke, T. L., et al. 2013, ApJ, 766, 115

Koyama H., \& Inutsuka S. I., 2000 ApJ, 532, 980

Koyama, H., \& Inutsuka, S.-I. 2002, ApJ, 564, L97

Kritsuk, A. G., Norman, M. L., \& Wagner, R. 2011, ApJ, 727, L20 
Kroupa, P. 2001, MNRAS, 322, 231

Krumholz, M. R., Dekel, A., \& McKee, C. F. 2012, ApJ, 745, 69

Krumholz, M. R., \& McKee, C. F. 2005, ApJ, 630, 250

Krumholz, M. R., McKee, C. F., \& Tumlinson, J. 2009, ApJ, 699, 850

Krumholz, M. R., \& Tan, J. C. 2007, ApJ, 654, 304

Lada, J. L., Lombardi, M., \& Alves, J. F. 2010, ApJ, 724, 687

Larson, R. B. 1985, MNRAS, 214, 379

Mac Low, M. -M. \& Klessen, R. S. 2004, Rev. Mod. Phys., 76, 125

McKee, C. F., \& Ostriker, E. C. 2007, ARA\&A, 45, 565

Micic, M., Glover, S. C. O., Banerjee, R., \& Klessen, R. S. 2013, MNRAS, 432, 626

Molinari, S., Swinyard, B., Bally, J., et al. 2010, A\&A, 518, L100

Mouschovias, T. C. 1991, NATO ASIC Proc. 342: The Physics of Star Formation and Early Stellar Evolution, 449

Myers P. C., 2009, ApJ, 700, 1609

Ostriker, E. C., Gammie, C. F., \& Stone, J. M. 1999, ApJ, 513, 259

Padoan, P., \& Nordlund, A., 2011, ApJ, 730, 40

Padoan, P., Nordlund, A., \& Jones, B. J. T. 1997, MNRAS, 288, 145

Palla, F., \& Stahler, S. W. 1999, ApJ, 525, 772

Palla, F., \& Stahler, S. W. 2000, ApJ, 540, 255 
Palla, F., \& Stahler, S. W. 2002, ApJ, 581, 1194

Passot, T. \& Vázquez-Semadeni, E. 1998, Phys. Rev. E, 58, 4501

Peretto, N., Hennebelle, P., \& André, P. 2007, A\&A, 464, 983

Peretto, N., Fuller, G. A., André, P., et al. 2014, A\&A, 561, A83

Pon, A., Toalá, J. A., Johnstone, D., et al. 2012, ApJ, 756, 145

Pound, M. W., \& Goodman, A. A. 1997, ApJ, 482, 334

Preibisch, T., \& Mamajek, E. 2008, Handbook of Star Forming Regions, Volume II, 235

Rosolowsky, E., Engargiola, G., Plambeck, R., \& Blitz, L. 2003, ApJ, 599, 258

Schmidt, M. 1959, ApJ, 129, 243

Schneider, N., Csengeri, T., Bontemps, et al. 2010, A\&A, 520, A49

Shu, F. H., Adams, F. C., \& Lizano, S. 1987, ARA\&A, 25, 23

Smith, R. J., Longmore, S., \& Bonnell, I. 2009, MNRAS, 400, 1775

Tan, J. C., Beltran, M. T., Caselli, P., et al. 2014, arXiv:1402.0919

Toalá, J. A., Vázquez-Semadeni, E., \& Gómez, G. C. 2012, ApJ, 744, 190

Vázquez-Semadeni, E. 1994, ApJ, 423, 681

Vázquez-Semadeni, E., Ballesteros-Paredes, J., \& Klessen, R. S. 2003, ApJ, 585, L131

Vázquez-Semadeni, E., Banerjee, R., Gómez G. C., Hennebelle, P., Duffin, D. \& Klessen, R. S. 2011, MNRAS, 414, 2511

Vázquez-Semadeni, E., Colín, P., Gómez, G. C., Ballesteros-Paredes, J., \& Watson, A. W. 2010, ApJ, 715, 1302 
Vázquez-Semadeni, E., Gómez, G. C., Jappsen, A. K., Ballesteros-Paredes, J., González, R. F., \& Klessen, R. S. 2007, ApJ, 657, 870

Vázquez-Semadeni, Gómez, G. C., Jappsen, A.-K., Ballesteros-Paredes, J. \& Klessen, R. S. 2009, ApJ, 707, 1023

Vázquez-Semadeni, E., González, R. F., Ballesteros-Paredes, J., Gazol, A., \& Kim, J. 2008, MNRAS, 390, 769

Vazquez-Semadeni, E., Ostriker, E. C., Passot, T., Gammie, C. F., \& Stone, J. M. 2000, Protostars and Planets IV, 3

Vázquez-Semadeni, E., Ryu, D., Passot, T., González, R. F., \& Gazol, A. 2006, ApJ, 643, 245

Vishniac E. T. 1994, ApJ, 428, 186

Walder, R., \& Folini, D. 2000, ApSS, 274, 343

Williams JP, \& McKee C. F. 1997. ApJ, 476, 166

Wu, J., Evans, N. J., Shirley, Y. L., \& Knez, C. 2010, ApJS, 188, 313

Zuckerman B., \& Evans N. J. 1974, ApJ, 192, L149

Zuckerman, B., \& Palmer, P. 1974, ARA\&A, 12, 279

Zamora-Avilés , M. A., Vázquez-Semadeni, E., \& Colín, P. 2012, ApJ, 751, 77 